\title{
Liquid marble containing degradable polyperoxides for adhesion force-changeable pressure-sensitive adhesives
}

\author{
Eriko Sato, Michihiro Yuri, Syuji Fujii, Takashi Nishiyama, \\ Yoshinobu Nakamura and Hideo Horibe
}

\begin{tabular}{|c|l|}
\hline Citation & Rsc Advances, 6(61); 56475-56481 \\
\hline Issue Date & 2016-06 \\
\hline Type & Journal Article \\
\hline Textversion & author \\
\hline $\begin{array}{c}\text { Supplementary } \\
\text { files }\end{array}$ & Supplementary information is available at https://doi.org/10.1039/C6RA10677C \\
\hline Rights & $\begin{array}{l}\text { The following article has been accepted by Rsc Advances. After it is published, it } \\
\text { will be found at https://doi.org/10.1039/C6RA10677C }\end{array}$ \\
\hline DOI & $10.1039 /$ C6RA10677C \\
\hline
\end{tabular}

\author{
Self-Archiving by Author(s) \\ Placed on: Osaka City University
}

SATO, E., YURI, M., FUJII, S., NISHIYAMA, T., NAKAMURA, Y., \& HORIBE, H. (2016). Liquid marble containing degradable polyperoxides for adhesion force-changeable pressure-sensitive adhesives. RSCAdvances. 6, 61, 56475-56481. https://doi.org/10.1039/C6RA10677C 


\section{Journal Name}

\section{ARTICLE}

\section{Liquid Marble Containing Degradable Polyperoxides for Adhesion Force-Changeable Pressure-Sensitive Adhesives}

Received 00th January 20xx Accepted 00th January 20xx

DOI: $10.1039 / x 0 x \times 00000 x$

\author{
Eriko Sato*, ${ }^{a}$ Michihiro Yuri, ${ }^{a}$ Syuji Fujii, ${ }^{b}$ Takashi Nishiyama, ${ }^{a}$ Yoshinobu Nakamura, ${ }^{b}$ and Hideo \\ Horibe $^{\text {a }}$
}

\begin{abstract}
Liquid marbles containing a sticky polyperoxide were prepared by the in-situ copolymerization of the 1,3-diene monomer with oxygen. The as-prepared liquid marbles had a non-sticky nature and could move on any substrates due to the presence of hard particles on their surfaces, while the squeezed liquid marble developed adhesion force-tunable PSA properties due to the outflow of the inner materials. The $180^{\circ}$ peel strength of the squeezed liquid marble increased by heating at $90{ }^{\circ} \mathrm{C}$ for $1 \mathrm{~h}$ or by UV irradiation at $0.86 \mathrm{~J} / \mathrm{cm}^{2}$ because the cohesive force of the adhesive layer increased by the additional polymerization initiated by moderate decomposition of the polyperoxide. The increased $180^{\circ}$ peel strengths were significantly decreased again to almost zero, i.e., dismantling, after an additional heating at $150{ }^{\circ} \mathrm{C}$ for $1 \mathrm{~h}$ because of an excess increase in the cohesive force as pressure-sensitive adhesives, where cross-linking took place as a result of significant decomposition of the polyperoxide accompanied by hydrogen abstraction and coupling.
\end{abstract}

\section{Introduction}

Polyperoxides contain a peroxy bond as the main-chain repeating units and undergo main-chain decomposition by various stimuli such as heating, UV irradiation, and treatment with reductants. ${ }^{1,2}$ Due to the radical-forming properties of polyperoxides similar to low molecular weight organic peroxides, polyperoxides are used as macroinitiators for the radical polymerization of vinyl monomers. ${ }^{3,4}$ In particular, polyperoxides undergo decomposition under mild conditions, such as heating around $100{ }^{\circ} \mathrm{C}$, and have attracted attention as a new type of degradable polymeric material. Conventional organic materials are generally stable at the temperature at which the polyperoxides undergo thermal decomposition, and the polyperoxides can be selectively decomposed in composite materials. Due to their readily degradable nature, polyperoxides are also utilized as a degradable linker for graft and network polymers based on vinyl polymers, biodegradable polylactide, and biocompatible polyethylene glycol. ${ }^{5-7}$ Furthermore, polyperoxides and their derivatives, including branched and block copolymers, have been applied to various types of degradable functional materials, i.e., dismantlable (de-bondable) adhesives, ${ }^{8,9}$ coatings for surface modification, ${ }^{9,10}$ and biocompatible materials. $^{11,12}$ Matsumoto et al. reported that the radical alternating

\footnotetext{
a. Department of Applied Chemistry and Bioengineering, Graduate School of Engineering, Osaka City University, 3-3-138 Sugimoto, Sumiyoshi-ku, Osaka 5588585, Japan. E-mail: sato@a-chem.eng.osaka-cu.ac.jp

${ }^{b .}$ Department of Applied Chemistry, Faculty of Engineering, Osaka Institute of Technology, 5-16-1 Omiya, Asahi-ku, Osaka 535-8585, Japan

Electronic Supplementary Information (ESI) available: Details for the probe tack measurement, digital microscope images, ${ }^{1} \mathrm{H}$ NMR, the sliding experiment of the polymerized liquid marble, and photographs of the $180^{\circ}$ peel test specimens. See DOI: $10.1039 / x 0 x \times 00000 x$
}

copolymerization of 1,3-diene monomers with oxygen smoothly proceeded in solution under oxygen bubbling, i.e., at atmospheric pressure, in good yields. ${ }^{13}$ Considering the fact that the synthesis of polyperoxides using general vinyl monomers requires a high oxygen pressure to increase the yield, ${ }^{1}$ the synthesis of polyperoxides using 1,3-diene monomers is versatile, facile, and suitable for practical applications.

Liquid marbles are core-shell type particles consisting of an inner liquid droplet with a high surface energy and outer solid particles with a low surface energy, and the liquid droplet is stabilized by the solid particles adsorbed on the surface. ${ }^{14-19}$ Although the stability of the liquid marbles depends on various factors, the inner liquid droplet of a liquid marble can often be transferred without wetting the substrates, and this unique feature has opened the way for broad applications including cosmetics, ${ }^{20,21}$ miniature reactors, ${ }^{22-24}$ personal \& health care products, ${ }^{25}$ sensors, ${ }^{26-}$ ${ }^{30}$ accelerometers, ${ }^{31}$ gas storage, ${ }^{32}$ transport \& microfluidics, ${ }^{33-35}$ shape-tunable freezing water, ${ }^{36}$ and pressure-sensitive adhesives (PSAs). ${ }^{37}$

We recently reported that liquid marbles act as a novel microreactor to efficiently synthesize polyperoxides from 1,3-diene monomers. ${ }^{38}$ The synthesis of polyperoxides in the liquid marbles does not require oxygen bubbling and stirring during the polymerization due to an adequate oxygen supply through the large and permeable gas-liquid interface, and has the potential to save manufacturing energy. As mentioned above, we previously reported that polyperoxides act as dismantlable adhesives and the polyperoxides derivatives with low glass transition temperature, i.e., sticky polyperoxides, show PSA behavior with dismantlable properties. ${ }^{8,9}$ Considering that the liquid marbles containing sticky inner materials are not sticky because of the presence of the outer hard solid particles ${ }^{37}$ we expected that the liquid marbles containing 
(a)

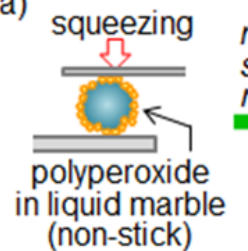

releasing sticky inner
materials

(b)

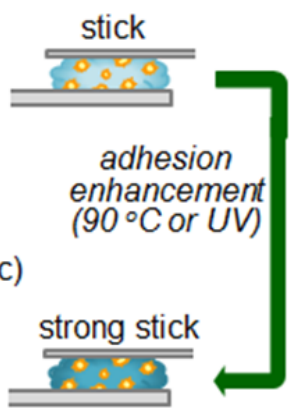

Fig. 1. Schematic illustration of the bonding of solid substrates using a liquid marble containing polyperoxides; (a) a non-stick liquid marble on the substrates, (b) the bonding of the substrates by squeezing of the liquid marble, (c) the bonding of the substrates with a strong stick by the adhesion enhancement treatment, and (d) the debonding of the substrates by the dismantling treatment.

sticky polyperoxides have the prospect to be a new type of dismantlable PSA, which does not require a solvent-coating and drying process and can be used without a support material and a release liner (Fig. 1). Dismantlable adhesives show a sufficient adhesion strength and can reduce the adhesion strength in response to external stimuli on demand, ${ }^{8,9,39-46}$ and were practically used for a temporary bonding and material recycling aimed at energy and resource savings. ${ }^{47-50}$ The application of the liquid marble containing a sticky polyperoxide as a dismantlable PSA can provide full energy and resource saving processes in terms of the synthesis of the PSA polymers, PSA processing, and the final product. Although energy and resource savings have been a crucial aim of dismantlable adhesion techniques, most research studies have focused on one of the synthetic procedures, materials, processing, and final products.

In this study, the liquid marbles containing a sticky polyperoxide, i.e., the polyperoxide from 2-hydroxyethyl sorbate (PP-HES), were prepared by the in-situ copolymerization of 2-hydroxyethyl sorbate (HES) with oxygen in the liquid marbles. The adhesion properties of the resulting liquid marbles were extensively investigated considering the application to adhesion force-tunable PSAs having a dismantlability. The reactions accompanying the decomposition of PP-HES in the liquid marbles were investigated to reveal the relationship between the chemical reactions and PSA properties.

\section{Experimental Section}

HES was prepared according to the method described in the literature. $^{51} \quad$ 2,2'-Azobis(4-methoxy-2,4-dimethylvaleronitrile) (AMVN) was recrystallized from methanol. Commercially available lycopodium (Sigma-Aldrich) was used as received. All other commercial chemicals were used as received without further purification.

\section{Polymerization of HES in a Liquid Marble}

The synthesis of PP-HES in a liquid marble was performed according to our previous report. ${ }^{38}$ A typical polymerization procedure is as follows. The ground AMVN crystals by a mortar were added to HES and the mixture was stirred at room temperature for $15 \mathrm{~min}$. A $15 \mu \mathrm{L}$ aliquot of the mixture was dropped on to the lycopodium powder in a desiccator, then rolled over on the powder to form the liquid marble. The desiccator was sealed and connected to an oxygen balloon. The liquid marble containing the mixture of HES and AMVN was polymerized at $40{ }^{\circ} \mathrm{C}$ for $48 \mathrm{~h}$ under the oxygen atmosphere. After the polymerization, the reaction mixture was dissolved in acetone- $d_{6}$ and subjected to ${ }^{1} \mathrm{H}$ NMR spectroscopy.

\section{Measurements}

The ${ }^{1} \mathrm{H}$ NMR spectra were recorded by a Bruker AV300N spectrometer. The differential scanning calorimetric (DSC) analysis was performed using a Seiko EXSTAR6000 at the heating rate of 10 ${ }^{\circ} \mathrm{C} / \mathrm{min}$.

\section{Probe Tack Tests}

The tack of the polymerized liquid marbles was measured using a probe tack tester (TE-6002, Tester Sangyo, Saitama, Japan) with a stainless-steel (SUS 304) probe (5 mm diameter) at $23 \pm 1{ }^{\circ} \mathrm{C}$. A polymerized liquid marble was placed on a probe, and a bobbin, which was bonded to a glass plate having a predetermined weight, was allowed to approach to the probe at $10 \mathrm{~mm} / \mathrm{min}$ (Fig. S1, ESI). The bobbin was held for $30 \mathrm{sec}$ while being lifted by the probe, then released from the probe at $10 \mathrm{~mm} / \mathrm{min}$ to $c a .4 .5 \mathrm{~mm}$ away from the lifted position. The probe tack measurements after the dismantling treatment were similarly performed by bonding the treated adhesive layer on the glass plate instead of placing it on the probe.

\section{$180^{\circ}$ Peel Tests}

A SUS430 $\left(150 \times 50 \times 0.5 \mathrm{~mm}^{3}\right)$ plate was cleaned by ultrasonication in acetone for $15 \mathrm{~min}$, then in 2-propanol for $15 \mathrm{~min}$. To prepare an adhesive tape, three polymerized liquid marbles were pressure squeezed and applied to a PET support film (20 mm x 250 $\mathrm{mm}, 50 \mu \mathrm{m}$ thickness) of $10 \mathrm{~cm}$ length from one end. The adhesive tape was pressure bonded onto a SUS plate using a 2-kg hand roller by reciprocating 5 times. A $180^{\circ}$ peel test was carried out after the specimen was left to stand for over $30 \mathrm{~min}$ at room temperature. For the UV irradiation, the adhesive tape with the adhesive layer-side up was placed at a distance of $10 \mathrm{~cm}$ from the UV source (Toshiba SHL-100UVQ-2) at room temperature. The light intensity of 0.24 $\mathrm{mW} / \mathrm{cm}^{2}$ was measured by a UV power meter (ORC Manufacturing, UV-M03A) with a UV-25 optical receiver $(240-275 \mathrm{~nm})$. For the thermal treatment, the peel test piece was placed in a preheated oven for a given time and then naturally cooled to room temperature. The $180^{\circ}$ peel tests were performed according to "ASTM D3330, standard test method for peel adhesion of pressure-sensitive tape", using a SHIMADZU AGS-X universal testing machine, with a $1 \mathrm{kN}$ (at maximum) load cell. All the adhesion tests were performed at 20 ${ }^{\circ} \mathrm{C}$ at the peel rate of $300 \mathrm{~mm} / \mathrm{min}$.

\section{Results and Discussion}

Preparation of the Liquid Marble Containing the Polyperoxide 
Table 1. Composition of the inner materials of the liquid marbles.

\begin{tabular}{lcccc}
\hline \multirow{2}{*}{ treatment } & \multicolumn{4}{c}{ Composition $^{a}(\mathrm{wt} \%)$} \\
\cline { 2 - 5 } & PP-HES & PHES & HES & FA-2HE \\
\hline untreated & 52 & 20 & 25 & 3 \\
$\mathrm{UV}\left(0.86 \mathrm{~J} / \mathrm{cm}^{2}\right)$ & 57 & 25 & 15 & 3 \\
\hline
\end{tabular}

${ }^{a}$ Determined by ${ }^{1} \mathrm{H}$ NMR analysis.

The liquid marbles consisting of HES as the inner liquid and lycopodium as the outer solid particles were prepared and the copolymerization of HES with oxygen was conducted at $40{ }^{\circ} \mathrm{C}$ for $48 \mathrm{~h}$. The diameter of the polymerized liquid marble prepared using $15 \mu \mathrm{L}$ of HES was approximately $3.5 \mathrm{~mm}$ and the average weight of the polymerized liquid marble was $16.8 \mathrm{mg}$. Both before and after the polymerization, the lycopodium powders were randomly absorbed on the liquid marble surface with a multilayer structure and a number of voids were observed in the lycopodium layer (Fig. S2, ESI). The chemical composition of the inner materials in the liquid marble was determined by a ${ }^{1} \mathrm{H}$ NMR analysis. As we previously reported, ${ }^{38}$ PP-HES was obtained as the main component (52 wt $\%$ ) and the rest consisted of PHES, unreacted HES, and one of the degradation products of PP-HES, fumaraldehyde 2-hydroxyethyl ester (FA-2HE) (Table 1). The loading amount of the outer lycopodium powder based on the inner materials was gravimetrically estimated to be less than $5 \mathrm{wt} \%$, in which the weight of the absorbed oxygen to form PP-HES was calculated from the resulting PP-HES content.

Thermal Degradation Behavior of PP-HES in the Liquid Marble The thermal degradation behavior of PP-HES, which was synthesized by solution polymerization, was investigated in our

(a)

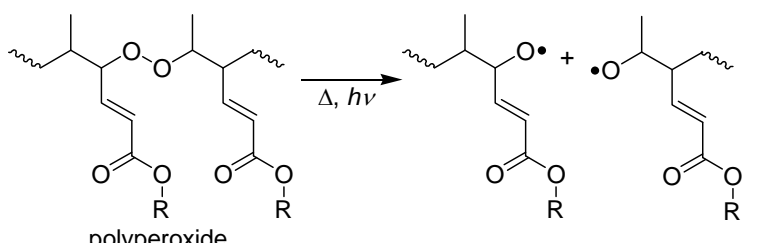

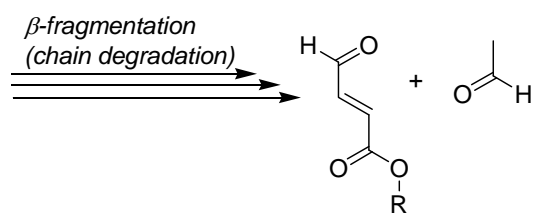

(b)

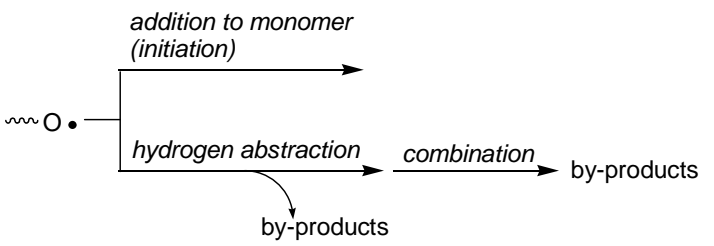

Scheme 1. (a) Decomposition mechanism of polyperoxides and (b) side-reactions of oxygen-centered radicals. previous study. ${ }^{51}$ It was reported that PP-HES showed a similar thermal degradation behavior to the polyperoxides from alkyl sorbate as shown in Scheme 1(a), and the onset degradation temperature was $102{ }^{\circ} \mathrm{C}$ and an exothermic peak in the differential thermal analysis curve was observed at $142{ }^{\circ} \mathrm{C}$ for PP-HES.

In order to evaluate the thermal degradation behavior of PP-HES in a liquid marble where PHES and HES also exist as by-products, the liquid marble was heated at $100{ }^{\circ} \mathrm{C}$ for $1 \mathrm{~h}$ as the temperature near the onset degradation temperature or at $150{ }^{\circ} \mathrm{C}$ for $1 \mathrm{~h}$ as the temperature near the peak top temperature of the exotherm for the PP-HES. The ${ }^{1} \mathrm{H}$ NMR analysis of the liquid marble after heating at $100{ }^{\circ} \mathrm{C}$ for $1 \mathrm{~h}$ showed an obvious decrease in the PP-HES content and an increase in the contents of the decomposition products, such as FA-2HE, as expected from the decomposition mechanism of the polyperoxides $^{38,52}$ (Scheme 1(a), Fig. S3, ESI). In addition to the decomposition of PP-HES, the polymerization of HES was indicated by the decreased HES content and the increased PHES content. Polyperoxides are known to act as macroinitiators ${ }^{53}$ and it is expected that the radicals generated by the decomposition of PPHES initiated the polymerization of HES in competition with the chain-decomposition of the oxygen-centered radicals (Scheme 1). It should be mentioned here that the radical initiator used for the preparation of the polymerized liquid marbles, i.e., AMVN, was all consumed during 48-h polymerization considering the half-life of AMVN (= ca. $175 \mathrm{~min}$, calculated by the decomposition rate constant of AMVN at $40{ }^{\circ} \mathrm{C}=6.6 \times 10^{-5} \mathrm{~s}^{-1} ; k_{\mathrm{d}}\left[\mathrm{s}^{-1}\right]=1.36 \times$ $\left.10^{16} \exp (-115 \quad[\mathrm{~kJ} / \mathrm{mol}] / \mathrm{RT})\right) .^{54} \quad$ Unfortunately, a quantitative discussion about the products after heating at $100{ }^{\circ} \mathrm{C}$ is difficult due to the complexities of the ${ }^{1} \mathrm{H}$ NMR spectrum. The side reactions of the oxygen-centered radicals, such as hydrogen abstraction and the combination of the resulting carbon-centered radicals, are expected to generate various by-products (Scheme 1(b)). After heating at $150{ }^{\circ} \mathrm{C}$ for $1 \mathrm{~h}$, the inner polymer component of the liquid marble became insoluble in any of the solvents used for the NMR measurement, thus the chemical compositions could not be determined. This result suggests that the significant decomposition of PP-HES produced frequent side reactions and thus the formation of cross-linked by-products at $150{ }^{\circ} \mathrm{C}$. The analysis of the thermal degradation behavior of the polyperoxides in the liquid marble revealed that the moderate decomposition of PP-HES at $100{ }^{\circ} \mathrm{C}$ results in the further polymerization of HES, and the significant
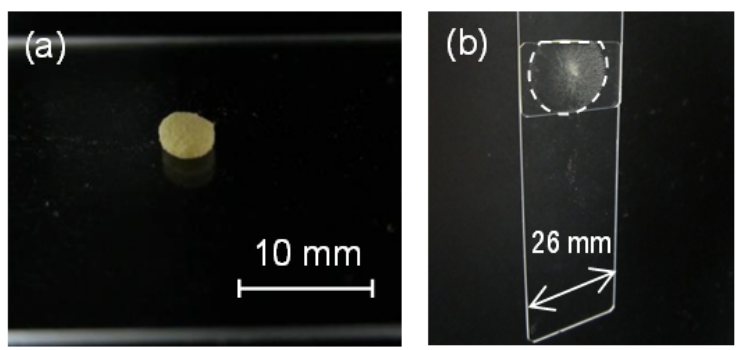

Fig. 2. Photographs of (a) the liquid marble containing PP-HES on a glass plate and (b) the glass plates bonded by a pressuresqueezed liquid marble. The surrounded area by the dashed curve shows the adhered area by the crushed liquid marble. 


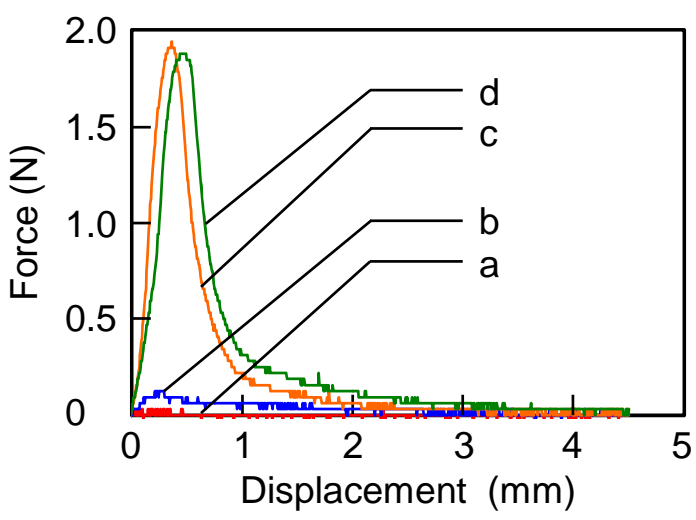

Fig. 3. Force-displacement curves obtained for the liquid marbles using the probe tack tester at various loadings: $2(\mathrm{a},-)$, $4(\mathrm{~b},-), 10(\mathrm{c},-)$, and $20 \mathrm{~g}(\mathrm{~d},-)$.

decomposition of PP-HES at $150{ }^{\circ} \mathrm{C}$ produces cross-linking.

\section{Adhesion Properties: Tack Properties}

Although the inner materials of the liquid marble were sticky because the glass transition temperature of PP-HES and PHES were relatively low ( -4.2 and $25{ }^{\circ} \mathrm{C}$, respectively), the polymerized liquid marble was stable and readily-transferable onto solid substrates such as a glass plate (Fig. 2(a)). The liquid marble placed on an inclined glass tube at approximately $30^{\circ}$ rolled down without sticking on the glass (Fig. S4, ESI). When the liquid marble sandwiched between the two glass plates was squeezed by a finger, the liquid marble was crushed and the sticky inner materials were released to immediately bond the glass plates to each other (Fig. 2(b)). The $4.5 \mathrm{~g}$ glass plate was able to be held by the one crushed liquid marble. This observation shows that the liquid marble behaves as a PSA which does not require neither a support film nor a release liner.

The relationship between the applied force and the adhesion strength was investigated using a probe tack tester. A bobbin bonded to a glass plate having different weights was approached to the probe, on which the liquid marble was placed, and the forces required to detach the bobbin from the probe were recorded (Fig. S1, ESI). Fig. 3 shows the force-displacement curves obtained for the bobbins weighing $2,4,10$, and $20 \mathrm{~g}$. The values of the maximum

Table 2. Results of the adhesion test of the liquid marble using the probe tack tester.

\begin{tabular}{cccc}
\hline $\begin{array}{c}\text { load } \\
(\mathrm{g})\end{array}$ & $\begin{array}{c}\text { maximum force } \\
(\mathrm{N})\end{array}$ & $\begin{array}{c}\text { adhesion energy } \\
(\mathrm{mJ})\end{array}$ & remarks $^{a}$ \\
\hline $2(1)^{b}$ & $0.03(1.5)^{b}$ & $\sim 0$ & $\mathrm{~A}$ \\
$4(2)^{b}$ & $0.13(6.6)^{b}$ & 0.13 & $\mathrm{~A}$ \\
$10(5)^{b}$ & $1.94(99.0)^{b}$ & 1.02 & $\mathrm{~B}$ \\
$20(10)^{b}$ & $1.88(95.9)^{b}$ & 1.19 & $\mathrm{~B}$ \\
\hline
\end{tabular}

${ }^{a} \mathrm{~A}$ : The surface of the crushed liquid marble was covered by lycopodium. B: Lycopodium was buried in the sticky inner materials. ${ }^{b}$ The values in the parentheses denote the stress in $\mathrm{kPa}$ assuming the full contact of the crushed liquid marble and the substrates. (a)

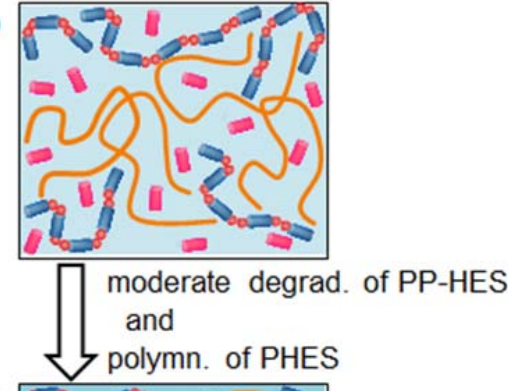

(b)
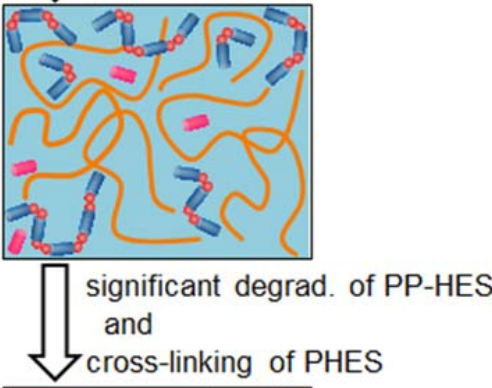

(c)

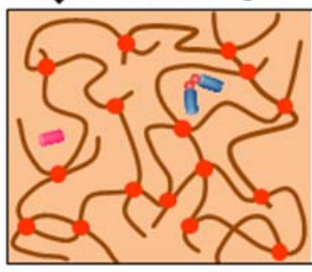

$=:$ HES

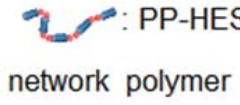

Fig. 4. Schematic illustration of the inner materials in the liquid marbles as prepared (a), after heating at $90{ }^{\circ} \mathrm{C}$ for $1 \mathrm{~h}(\mathrm{~b})$, and after subsequent heating at $150{ }^{\circ} \mathrm{C}$ for $1 \mathrm{~h}$.

force and the area of the curves, which describe the tack and adhesion energy, respectively, are summarized in Table 2. The values of the applied stress and the maximum stress are also shown in the parentheses as complementary information, because the liquid marble did not completely contact with the probe, which is usual phenomena for common PSAs. ${ }^{37}$ The maximum force and adhesion energy were very low up to $4 \mathrm{~g}$ of load, although the liquid marbles were crushed. The maximum force and adhesion energy significantly increased with the 10 and $20 \mathrm{~g}$ loads. When the load was less than $4 \mathrm{~g}$, the surface of the crushed liquid marble was covered by lycopodium, which was used as the hydrophobic solid particles to prepare the liquid marbles. The existence of the nonstick lycopodium at the interface of the PSA and the substrate should avoid full contact between the PSA and the substrate, resulting in very low maximum force and adhesion energy. On the other hand, lycopodium was buried in the sticky inner materials above a $10 \mathrm{~g}$ load. These results show that the sticky inner materials exited from the inside to cover the non-sticky outer solid particles just by crushing the liquid marble with a certain load.

\section{Adhesion Properties: Peel Properties}

The PSA properties of the crushed liquid marble were further 
Table 3. Results of the $180^{\circ}$ peel test after various treatments.

\begin{tabular}{lcccc}
\hline treatment & $\begin{array}{c}\text { peel strength } \\
(\mathrm{N} / 20 \mathrm{~mm})\end{array}$ & relative value & failure mode & solubility $^{a}$ \\
\hline untreated & 0.088 & - & cohesive & soluble \\
\hline $90^{\circ} \mathrm{C}(1 \mathrm{~h})$ & $5.9 \pm 0.99$ & 1 & cohesive & soluble \\
$90{ }^{\circ} \mathrm{C}(1 \mathrm{~h})+150{ }^{\circ} \mathrm{C}(1 \mathrm{~h})$ & 0.053 & $0.009^{b}$ & PET interfacial & insoluble \\
$\mathrm{UV}\left(0.86 \mathrm{~J} / \mathrm{cm}^{c}\right)$ & $2.7 \pm 1.6$ & 1 & cohesive & soluble \\
$\mathrm{UV}\left(0.86 \mathrm{~J} / \mathrm{cm}^{2}\right)+150^{\circ} \mathrm{C}(1 \mathrm{~h})$ & 0.021 & $0.008^{b}$ & PET interfacial $^{c}$ & insoluble \\
\hline
\end{tabular}

${ }^{a}$ The solubility of the adhesive layer in acetone. ${ }^{b}$ The peel strength relative to that before heating at $150{ }^{\circ} \mathrm{C}$ for $1 \mathrm{~h}$. ${ }^{c}$ Spontaneous failure was partially observed.

investigated in detail by the $180^{\circ}$ peel test. As summarized in Table 3 , the simply crushed liquid marble (untreated one) produced a very weak $180^{\circ}$ peel strength with a cohesive failure, in which the adhesive layer was broken off and remained on both the PET film and SUS plate substrate used for the peel test, i.e., cohesive failure. The peel behavior is known to be determined by the cohesive force of the adhesive layer and the interfacial interaction between the adhesive layer and the substrates, i.e., the adhesion force. When the cohesive force is weaker than the adhesion force, cohesive failure takes place and the peel strength is determined by the cohesive force. On the other hand, when the cohesive force is stronger than the adhesion force, interfacial failure between the adhesive layer and the substrate takes place and the peel strength is determined by the adhesion force. The current result reveals that the inner materials of the liquid marbles well wet both the PET film and the SUS plate substrate resulting in an adequate adhesion force, and the cohesive force of the inner materials, i.e., the adhesive layer, is low. The fact that the inner materials contain a certain amount of HES, which would act as a plasticizer, seems to be one of the major reasons for the low cohesive force. As already described, the decomposition of PP-HES takes place during heating of the liquid marbles, and the generated radicals initiate the polymerization of HES. It is expected that the decomposition of PP-HES in the adhesive layer similarly initiates the polymerization of HES. In order to consume HES in the

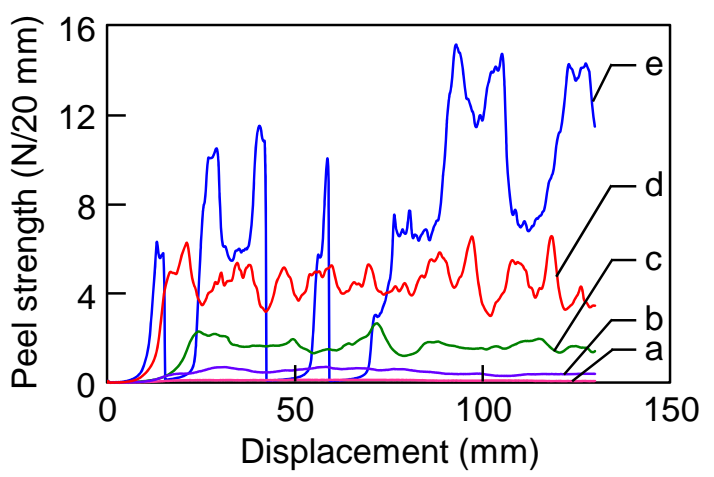

Fig. 5. The representative peel strength-displacement curves for the $180^{\circ}$ peel test of the liquid marbles: after heating at $60(\mathrm{a}$, $-), 70(\mathrm{~b},-), 80(\mathrm{c},-), 90(\mathrm{~d},-)$, and $100^{\circ} \mathrm{C}(\mathrm{e},-)$ for $1 \mathrm{~h}$. adhesive layer by polymerization (Fig. 4(a) and (b)), the $180^{\circ}$ peel test specimens were heated at a given temperature for $1 \mathrm{~h}$. Fig. 5 shows the representative peel strength-displacement curves after heating. The peel strength increased with an increase in the heating temperature from 60 to $90{ }^{\circ} \mathrm{C}$ and successfully reached $5.9 \pm 0.99$ $\mathrm{N} / 20 \mathrm{~mm}$ (Table 3). The value of the $180^{\circ}$ peel strength is comparable to that of a commercially available mending tape, which resulted in $5.4 \mathrm{~N} / 20 \mathrm{~mm}$ under the same peel test conditions. As seen in Fig. 5, heating at $100{ }^{\circ} \mathrm{C}$ brought about an unsteady peel strength because of the void formation probably due to the elimination of volatile acetaldehyde ${ }^{9}$ and/or increased fluidity of the adhesive layer by heating (Fig. S5, ESI). The $180^{\circ}$ peel strength was also raised by UV irradiation $\left(0.86 \mathrm{~J} / \mathrm{cm}^{2}\right)$ of the adhesive layer at room temperature (Table 3). The ${ }^{1} \mathrm{H}$ NMR analyses of the adhesive layer demonstrated that the consumption of HES and the formation of PP-HES and PHES by UV irradiation (Table 1). It is postulated that the consumption of HES and the formation of polymers synergistically increased the cohesive force of the adhesive layer. We confirmed that an approximately $5 \mathrm{wt} \%$ difference in the composition of the adhesive materials resulted in a considerable difference in the $180^{\circ}$ peel behavior using the separately prepared PP-HES and PHES (Table S1, ESI). The conversion of HES to the polymers successfully increased the $180^{\circ}$ peel strength of the squeezed liquid marbles. The treatments, such as heating at $90{ }^{\circ} \mathrm{C}$ and UV irradiation, are regarded as "adhesion enhancement treatments".

\section{Adhesion Properties: Dismantlable Behavior}

It is known that the modulus of PSA materials is required to be in a certain range to have good PSA properties. ${ }^{47,55}$ In other words, both a "too low" or "too high" modulus results in a weak peel strength, thus it is expected that the formation of network structures, which increases the modulus, ${ }^{47}$ brings about a deterioration in the peel strength. The decomposition of PP-HES at $150{ }^{\circ} \mathrm{C}$ in the liquid marbles resulted in cross-linking as already described. The adhesive layers after the adhesion enhancement treatments still contain PPHES as the main component as shown in Table 1, and therefore, further significant decomposition of PP-HES is supposed to induce cross-linking (Figs. 4(b) and (c)).

The test specimens after the adhesion enhancement treatments, i.e., heating at $90{ }^{\circ} \mathrm{C}$ or UV irradiation, were further subjected to heating at $150{ }^{\circ} \mathrm{C}$ for $1 \mathrm{~h}$ as a dismantling treatment. As shown in 
Table 3 , in both cases, the values of the $180^{\circ}$ peel strength decreased to less than $1 \%$ of those achieved by the adhesion enhancement treatments. After the dismantling treatment, the adhesive layer turned into a non-sticky hard film-like material and insoluble in solvents such as chloroform and tetrahydrofuran. The failure mode changed from a cohesive failure to a PET interfacial failure by the dismantling treatment. The probe tack measurements of the adhesive layer after the dismantling treatment produced a very poor tack of less than $1.5 \mathrm{kPa}$ for the applied pressure from 5.1 to $100 \mathrm{kPa}$. These observations suggest that the modulus of the adhesive layer increased to a "too high" level, i.e., brittle, to be used as a PSA by heating at $150{ }^{\circ} \mathrm{C}$, and a significant decrease in the peel strength with an interfacial failure was achieved. We previously reported that the decomposition of polyperoxides resulted in a significant decrease in the peel strength, i.e., dismantling, due to the synergistically reduced cohesive force by the main-chain decomposition of the polyperoxides and the formation of low molecular weight degradation products which act as a plasticizer. ${ }^{8,9}$ In these cases, the failure modes after dismantling were cohesive failure and the decrease in the peel strength was due to a "too low" cohesive force of the adhesive layer. In this study, it was demonstrated that the decomposition of the polyperoxides induces not only a decrease in the cohesive force, ${ }^{8,9}$ but also an increase in the modulus by crosslinking. Although both can be a driving force to reduce the peel strength, the former produces cohesive failure and the later achieves interfacial failure. Taking into account the practical application of dismantlable adhesives, dismantling achieved by an increased modulus is preferable because the interfacial failure results in less contamination of the substrates. The present investigation revealed that liquid marbles containing sticky polyperoxides are promising materials as adhesion force-tunable PSAs having a dismantlability. Such multifunctional adhesive materials are useful in terms of green technology, i.e., a reduction of waste, energy savings, and environmentally-friendly processing.

\section{Conclusions}

We demonstrated that the liquid marble containing the degradable polyperoxide, PP-HES, as the main inner material behaves as an adhesion force-tunable PSA. The liquid marbles containing sticky inner materials did not show an adhesion on solid substrates and were movable. When the liquid marble was squeezed by more than $10 \mathrm{~g}$ of load, the inner materials were released to well cover the outer solid particles, and the adhesion properties were developed. Adhesion enhancement treatments, i.e., heating at $90{ }^{\circ} \mathrm{C}$ for $1 \mathrm{~h}$ or UV irradiation at $0.86 \mathrm{~J} / \mathrm{cm}^{2}$, significantly increased the $180^{\circ}$ peel strengths of the liquid marbles from almost zero to levels comparable to that of the commercially available PSA tape. This is because moderate decomposition of the polyperoxides in the liquid marble increased the cohesive force by consuming HES by polymerization. When the test specimens after the adhesion enhancement treatment were further subjected to heating at $150{ }^{\circ} \mathrm{C}$ for $1 \mathrm{~h}$ as the dismantling treatment, the $180^{\circ}$ peel strength decreased to less than $1 \%$ of the enhanced values. Significant decomposition of the polyperoxides at $150{ }^{\circ} \mathrm{C}$ resulted in cross-linking, and the peel strength decreased by an excessive increase in the modulus.
Appropriate treatments enable the tuning of the adhesion force of the liquid marbles. The liquid marbles containing sticky polyperoxides were proved to be promising materials as environmentally friendly and energy saving functional PSAs.

\section{Notes and references}

1. T. Mukundan and K. Kishore, Prog. Polym. Sci., 1990, 15, 475.

2. E. Sato and A. Matsumoto, Chem. Rec., 2009, 9, 247-257.

3. T. Mukundan, V. A. Bhanu and K. Kishore, J. Chem. Soc., Chem. Commun., 1989, 12, 780-781.

4. A. K. Nanda and K. Kishore, J. Polym. Sci.:Part A: Polym. Chem., 2000, 38, 3665-3673.

5. T. Kitamura and A. Matsumoto, Macromolecules, 2007, 40, 61436149.

6. T. Kitamura and A. Matsumoto, Macromolecules, 2007, 40, 509-517.

7. E. Sato, T. Kitamura and A. Matsumoto, Macromol. Rapid Commun., 2008, 29, 1950-1953.

8. E. Sato, H. Tamura and A. Matsumoto, ACS Appl. Mater. Interfaces, 2010, 2, 2594-2601.

9. E. Sato, T. Hagihara and A. Matsumoto, ACS Appl. Mater. Interfaces, 2012, 4, 2057-2064.

10. V. Samaryk, A. Voronov, I. Tarnavchyk, S. Varvarenko, N. Nosova, O. Budishevska, A. Kuhut and S. Voronov, Prog. Org. Coat., 2012, 74, 687-696.

11. T. Fujioka, S. Taketani, T. Nagasaki and A. Matsumoto, Bioconjugate Chem., 2009, 20, 1879-1887.

12. S. Pal, A. Das, S. Maiti and P. De, Polym. Chem., 2012, 3, 182-189.

13. A. Matsumoto and H. Higashi, Macromolecules, 2000, 33, 1651-1655.

14. P. Aussillous and D. Quere, Nature, 2001, 411, 924-927.

15. S. Fujii and R. Murakami, Kona Powder Particle J., 2008, 26, 153-166

16. P. Aussillous and D. Quéré, Proc. R. Soc. A, 2006, 462, 973-999.

17. E. Bormashenko, Curr. Opin. Colloid Interface Sci., 2011, 16, 266271.

18. G. McHale and M. I. Newton, Soft Matter, 2011, 7, 5473-5481.

19. G. McHale and M. I. Newton, Soft Matter, 2015, 11, 2530-2546.

20. WO Patent. 034917, 2005.

21. US Pat., US 62909412001.

22. Y. Xue, H. Wang, Y. Zhao, L. Dai, L. Feng, X. Wang and T. Lin, Adv. Mater., 2010, 22, 4814-4818.

23. T. Arbatan, A. Al-Abboodi, F. Sarvi, P. P. Y. Chan and W. Shen, Adv. Health. Mater., 2012, 1, 467-469.

24. D. Zang, J. Li, Z. Chen, Z. Zhai, X. Geng and B. P. Binks, Langmuir, 2015, 31, 11502-11507.

25. T. Arbatan, L. Li, J. Tian and W. Shen, Adv. Health. Mater., 2011, 1, 80-83.

26. E. Bormashenko and A. Musin, Appl. Surf. Sci., 2009, 255, 6429-6431.

27. J. Tian, T. Arbatan, X. Li and W. Shen, Chem. Commun., 2010, 46, 4734-4736.

28. V. Sivan, S.-Y. Tang, A. P. O'Mullane, P. Petersen, N. Eshtiaghi, K. Kalantar-zadeh and A. Mitchell, Adv. Funct. Mater., 2013, 23, 144152.

29. D. Dupin, S. P. Armes and S. Fujii, J. Am. Chem. Soc., 2009, 131, 5386-5387.

30. S. Yusa, M. Morihara, K. Nakai, S. Fujii, Y. Nakamura, A. Maruyama and N. Shimada, Polym. J., 2014, 46, 145-148.

31. H. Zeng and Y. Zhao, Appl. Phys. Lett., 2010, 96, 114104

32. W. Wang, C. L. Bray, D. J. Adams and A. I. Cooper, J. Am. Chem. Soc., 2008, 130, 11608-11609.

33. E. Bormashenko, R. Pogreb, Y. Bormashenko, A. Musin and T. Stein, Langmuir, 2008, 24, 12119-12122.

34. J. R. Dorvee, M. J. Sailor and G. M. Miskelly, Dalton Trans., 2008, 6, 721-730.

35. M. Paven, H. Mayama, T. Sekido, H.-J. Butt, Y. Nakamura and S. Fujii, Adv. Funct. Mater., 2016, 26, 3199-3206.

36. D. Zang, K. Lin, W. Wang, Y. Gu, Y. Zhang, X. Geng and B. P. Binks, Soft Matter, 2014, 10, 1309-1314.

37. S. Fujii, S. Sawada, S. Nakayama, M. Kappl, K. Ueno, K. Shitajima, H.-J. Butt and Y. Nakamura, Mater. Horiz., 2016, 3, 47-52.

38. E. Sato, M. Yuri, S. Fujii, T. Nishiyama, Y. Nakamura and H. Horibe, Chem. Commun., 2015, 51, 17241-17244. 
39. T. Inui, E. Sato and A. Matsumoto, ACS Appl. Mater. Interfaces, 2012, 4, 2124-2132.

40. T. Inui, K. Yamanishi, E. Sato and A. Matsumoto, Macromolecules, 2013, 46, 8111-8120.

41. T. Inui, E. Sato and A. Matsumoto, RSC Adv., 2014, 4, 24719-24728.

42. E. Sato, K. Yamanishi, T. Inui, H. Horibe and A. Matsumoto, Polymer, 2015, 64, 260-267.

43. M. Kobayashi and A. Takahara, Polym. Chem., 2013, 4, 4987-4992.

44. R. Wang, X. Xiao and T. Xie, Macromol. Rapid Commun., 2010, 31, 295-299.

45. S. Leijonmarck, A. Cornell, C.-O. Danielsson, T. Akermark, B. D. Brandner and G. Lindbergh, Int. J. Adhes. Adhes., 2012, 32, 39-45.

46. R. S. Gurney, D. Dupin, J. S. Nunes, K. Ouzineb, E. Siband, J. J. Asua S. P. Armes and J. L. Keddie, ACS Appl. Mater. Interfaces, 2012, 4, 5442-5452.

47. K. Ebe, H. Seno and K. Horigome, J. Appl. Polym. Sci., 2003, 90, 436441.

48. J. M. Boyne, E. J. Millan and I. Webster, Int. J. Adhes. Adhes., 2001, 21, 49-53.

49. H. Ishikawa, K. Seto, S. Shimotuma, N. Kishi and C. Sato, Int. J. Adhes. Adhes., 2005, 25, 193-199.

50. US Pat., US 2007/0269659 A1, 2007.

51. A. Mihashi, H. Tamura, E. Sato and A. Matsumoto, Prog. Org. Coat., 2010, 67, 85-91.

52. H. Hatakenaka, Y. Takahashi and A. Matsumoto, Polym. J., 2003, 35, 640-651.

53. A. K. Nanda and K. Kishore, Macromolecules, 2001, 34, 1558-1563.

54. K. W. Dixon, in Polymer Handbook, eds. J. Brandrup, E. H. Immergut and E. A. Grulke, John Wiley \& Sons, New York, 4th edn., 1999.

55. R. L. Patrick, ed., Treatise on Adhesion and Adhesives: Vol. 2: Materials, Marcel Dekker, New York, 1969. 